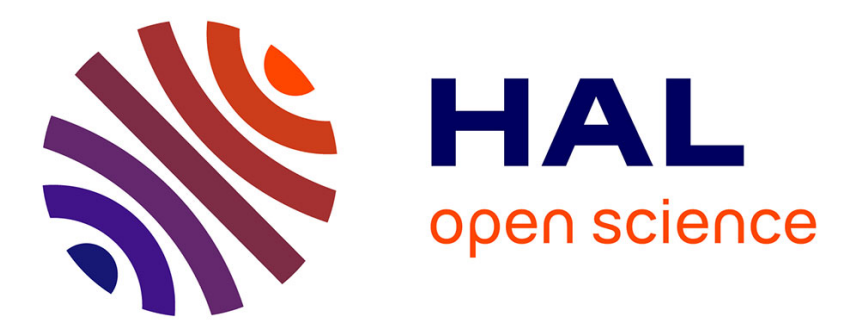

\title{
Electron angular distributions in near-threshold atomic ionization
}

T Marchenko, H G Muller, K J Schafer, M J J Vrakking

\section{To cite this version:}

T Marchenko, H G Muller, K J Schafer, M J J Vrakking. Electron angular distributions in nearthreshold atomic ionization. Journal of Physics B: Atomic, Molecular and Optical Physics, 2010, 43 (9), pp.95601. 10.1088/0953-4075/43/9/095601 . hal-00569916

\section{HAL Id: hal-00569916 https://hal.science/hal-00569916}

Submitted on 25 Feb 2011

HAL is a multi-disciplinary open access archive for the deposit and dissemination of scientific research documents, whether they are published or not. The documents may come from teaching and research institutions in France or abroad, or from public or private research centers.
L'archive ouverte pluridisciplinaire $\mathbf{H A L}$, est destinée au dépôt et à la diffusion de documents scientifiques de niveau recherche, publiés ou non, émanant des établissements d'enseignement et de recherche français ou étrangers, des laboratoires publics ou privés. 


\title{
Electron angular distributions in near-threshold atomic ionisation.
}

\author{
T Marchenko ${ }^{1,2,3}$, H G Muller ${ }^{1}$, K J Schafer ${ }^{4}$ and M J J Vrakking ${ }^{1}$ \\ ${ }^{1}$ FOM Institute for Atomic and Molecular Physics (AMOLF), Science Park 113, 1098XG \\ Amsterdam, The Netherlands \\ ${ }^{2}$ Laboratoire d'Optique Appliquée, UMR 7639 CNRS-ENSTA-Ecole Polytechnique, Chemin \\ de la Hunière, F-91761 Palaiseau, France \\ ${ }^{3}$ Laboratoire de Chimie Physique-Matière et Rayonnement, UMR 7614 UPMC Univ Paris 6- \\ CNRS, 11 rue Pierre et Marie Curie, F-75005 Paris, France \\ ${ }^{4}$ Department of Physics and Astronomy, Louisiana State University, Baton Rouge, Louisiana \\ 70803-4001, USA \\ E-mail: tatiana.marchenko@upmc.fr
}

\begin{abstract}
We present angle - and energy-resolved measurements of photoelectrons produced in strongfield ionisation of Xe using a tunable femtosecond laser. An occurrence of highly oscillatory patterns in the angular distribution at low photoelectron kinetic energy is observed that correlates with channel closing/opening over a wide range of laser parameters. The correlation is investigated both experimentally and by means of systematic analysis of numerical solutions of the time-dependent Schrödinger equation (TDSE). Our experimental and numerical results are in quantitative agreement with the semi-classical model introduced by Arbó et al. (Phys. Rev. A 78, 013406 (2008)), which relates the oscillatory patterns to interference between photoelectrons produced during different cycles of the laser pulse in the course of non-resonant ionisation of the atom. We observe that an increase of the laser intensity eventually leads to qualitative invariance of the pattern, defining a limit on the applicability of the semi-classical model.
\end{abstract}

(Some figures in this article are in colour only in the electronic version)

\section{Introduction}

Two- and three-dimensional photoelectron imaging techniques have recently become an important tool in investigations of strong-field light-matter interactions. The simultaneous observation of photoelectron kinetic energy and angular distributions in above-threshold ionisation (ATI) is a powerful tool for distinguishing different mechanisms that play a role in ionisation. For example, in strong-field ionisation of Xe [1] photoelectron imaging facilitated the identification of Rydberg states that were involved in resonance-enhanced ionisation. A simple relation was found between the angular momentum of these Rydberg states and the number of nodes that were observed in the continuum photoelectron angular distributions. 
In the last few years the kinetic energy region below the first ATI order has attracted a lot of attention. A radial fan-like pattern has been observed both experimentally [2,3] and in calculations [4, $5,6,7,8]$, and several theoretical attempts to explain the observed structures have been reported. Arbó et al. performed both time-dependent Schrödinger equation (TDSE) calculations and classical trajectory Monte-Carlo with tunnelling (CTMC-T) calculations for a hydrogen atom [4], and qualitatively reproduced the experimental results of Rudenko et al. 2]. A further analysis of the CTMC-T results led to the interpretation that the observed fan-like patterns are due to interference between different trajectories of the continuum photoelectrons in the combined laser and Coulomb field of the parent ion. In the calculation these trajectories are distinguished by the time of ionisation (at different peaks of the laser electric field) and consequently the number of quiver oscillations that the electron exhibits in the laser field. The trajectories are strongly influenced by the interaction of the electron with both the laser field and the ionic core. This sensitivity of the near-threshold radial pattern to the existence of a long-range Coulomb interaction was confirmed by Wickenhauser et al. [5] and Chen et al. [6], who performed a comparison between momentum maps resulting from numerical solution of the TDSE and from a model based on the Strong Field Approximation (SFA). In the latter paper, a number of different long range potentials were used, which included a variable cut-off function. An empirical rule was given for the dominant orbital angular momentum in the fan-like pattern, which was shown to be determined by the minimum number of photons needed to ionize the atom. However, no physical interpretation of this dominant angular momentum was provided. An interpretation was recently presented by Arbó et al. [8] in an elaboration of their earlier theoretical analysis. In this paper, electron momentum distributions resulting from TDSE and CTMC-T calculations were presented for Ar, supporting the notion that the near-threshold radial pattern can be quantitatively explained in terms of the properties of interfering classical trajectories in the presence of both the Coulomb and laser fields. According to the analysis of Arbó et al. the dominant angular momentum in the fan-like patterns is virtually independent of the atomic species. Rather, the classical electron angular momentum distribution peaks around a quantum number $l_{0}$, which depends on the laser field amplitude and frequency, or equivalently, the ionisation potential and the minimum number of photons needed to reach the continuum. This dependence was quantified in a semi-classical expression for $l_{0}$, that has been shown to provide quantitative agreement with the empirical relation proposed by Chen et al. [6] and previously published results of TDSE calculations [4, 5, 6, 9, 10].

In spite of the rather thorough theoretical description of the radial pattern appearing in the electron momentum spectra below the first ATI order, a comprehensive experimental study of this phenomenon has been lacking so far. In this paper we present experimental photoelectron momentum spectra for Xe over a wide range of intensities and wavelengths that allow the observation of qualitative variations in the radial pattern that are related to the occurrence of channel closing effects. Specifically, we present measurements at a wavelength of $700 \mathrm{~nm}$ over a range of intensities from $3.4 \times 10^{13} \mathrm{~W} / \mathrm{cm}^{2}$ to $9.5 \times 10^{13} \mathrm{~W} / \mathrm{cm}^{2}$ as well as measurements where the wavelength was varied 
between 600 and $800 \mathrm{~nm}$, while maintaining a peak intensity of appr. $46 \times 10^{13} \mathrm{~W} / \mathrm{cm}^{2}$. In addition, we performed extensive TDSE calculations for both Xe and Ar. A systematic analysis demonstrates quantitative agreement with the experiments and with the semi-classical expression for the dominant angular momentum proposed by Arbó et al. [8]. Furthermore, we observe that an increase of the laser intensity eventually leads to qualitative invariance of the pattern, defining a limit on the applicability of the semi-classical model.

\section{Experimental and theoretical methods}

In the experiment, a commercial laser system consisting of an optical parametric amplifier (OPA, Coherent Opera) pumped by $1 \mathrm{~mJ}$ from a $1 \mathrm{kHz}$ Ti:Sapphire amplifier system (800 nm, $50 \mathrm{fs}$ ) was used. The OPA signal output was frequency-doubled using a BBO crystal, providing wavelengths in the range from 600 to $800 \mathrm{~nm}$ with a FWHM of 10-15 nm. The central wavelength was determined with an accuracy of $2 \mathrm{~nm}$ using a spectrometer. The linearly polarised laser beam was focused onto a pulsed atomic beam $(25 \mathrm{~Hz})$ using a spherical normatincidence silver mirror $(\mathrm{f}=75 \mathrm{~mm})$. The ejected electrons were detected using a Velocity Map Imaging (VMI) spectrometer [11], consisting of a set of acceleration electrodes, a flight tube, a dual MCP and a phosphor screen. The DC field strength at the laser-atom interaction region was $600 \mathrm{~V} / \mathrm{cm}$ (appr. $10^{-7}$ a. u.) and its effect on the photoelectron spectra could be neglected in the presence of a strong laser field (appr. $3 \times 10^{-2}$ a. u.). Images were recorded using a computer-controlled CCD camera. Retrieval of the velocity and angular distribution of the measured photoelectrons was performed by using an iterative procedure of inverse Abel transform [12].

In order to explore the evolution of the momentum spectra with the laser intensity photoelectron momentum maps were recorded in Xe for a range of intensities at $700 \mathrm{~nm}$, i.e. using the maximum of the OPA efficiency curve. The laser pulse energy $E_{\text {pulse }}$ (as measured in the experiment) was varied between 25 and $60 \mu \mathrm{J}$ by inserting a neutral density filter in the path of the laser beam. Experimental images were obtained by summing the electron yield during one minute of acquisition time. In addition, images were recorded over a range of wavelengths between 600 and $800 \mathrm{~nm}$ while attempting to keep the laser intensity constant.

In what follows the actual intensity that was used in all measurements will be inferred by comparing the measured results to numerical solutions of the 3D TDSE within the single-active electron (SAE) approximation [13], i.e. considering only the response of the outermost valence electron to the laser field. In order to solve the TDSE for Xe we used a code based on the method outlined in $[14,15]$ with a pseudo-potential described in [16]. The TDSE for Ar was solved using the numerical algorithm described in [17]. Application of this algorithm for calculations of ATI spectra in a model Ar atom was previously demonstrated in [18]. Both codes provide energy-resolved electron angular distributions [19], which can be converted into a 2D momentum map that allows comparison with the experimentally recorded images. For an adequate comparison to the experiment the results of 
the TDSE calculations were integrated over the laser focal volume using the prescription described in [20], assuming a Gaussian focus in an infinitely extended medium. The temporal profile of the laser pulse was modeled with a sine-squared envelope typically containing 60 optical cycles. In our discussion, atomic units $\left(e=m_{e}=h=1\right)$ will be used throughout, unless mentioned otherwise.

\section{Results}

Figure 1 shows a series of slices through the 3D velocity and angular distribution of the ionised electrons (i.e. a momentum map) for ionisation of Xe at a $700 \mathrm{~nm}$ wavelength, where experimental images presented on the left are compared to TDSE results on the right. The vertical and horizontal axes in figure 1 show the electron momenta in a range from -0.4 to 0.4 a.u. parallel and perpendicular to the laser polarisation axis, respectively. This momentum scale corresponds to electron kinetic energies up to around $2.2 \mathrm{eV}$. The calculations are simulations of the experiment including full 3D volume averaging, assuming a laser focus up to an intensity specified by a value of the vector potential $A_{\max }=\left(I_{0} / \omega^{2}\right)^{1 / 2}=F_{0} / \omega$ where $I_{0}$ is the peak laser intensity and $F_{0}$ is the amplitude of the laser field. The peak laser intensity used in the calculations was chosen to provide the best agreement with the experimental data (based on visual inspection). Assignment of the matching pairs shows that the experiment covers a range of intensities from $3.4 \times 10^{13} \mathrm{~W} / \mathrm{cm}^{2}$ to $9.5 \times 10^{13} \mathrm{~W} / \mathrm{cm}^{2}$ with an estimated error of less than $5 \%$ in the individual intensities.

We distinguish two major contributions in the electron momentum spectra in figure 1. At values of the electron momentum higher than 0.2 a.u. the momentum maps are dominated by sharp oscillatory features, which can be attributed to $(n+1)$ resonance-enhanced ionisation via intermediate Rydberg states. A second contribution corresponds to electrons with an absolute value of the momentum below 0.2 a.u., i.e. a kinetic energy below $0.5 \mathrm{eV}$. This low-energy part of the photoelectron spectrum is characterised by highly oscillatory angular distributions and a continuous energy distribution extending down to zero.

The qualitative difference between these two contributions is reflected in their evolution with the laser intensity. Due to the fact that high-lying Rydberg states experience a ponderomotive Stark shift that is very similar to that of the ionisation threshold, ionisation via a selected resonant state will result in a photoelectron with the same kinetic energy independent of the laser intensity. An increase in the intensity will mainly be reflected in an increase of the ponderomotive energy and hence in the number of photons needed to resonantly populate a given Stark-shifted Rydberg state. A change in the number of photons required for resonant population of a Rydberg series is known as channel switching. Due to dipolar parity-selection rules channel switching is accompanied by an alternation between the excitation of odd- and even-parity Rydberg states and therefore manifests itself in changes in the photoelectron angular distribution. Monitoring the development of the resonance-induced feature at a kinetic energy of $1.2 \mathrm{eV}$ (indicated with a circle in figure 1a and $1 \mathrm{~g}$ ) as a function of intensity, we observe a qualitative change in the angular distribution around an intensity of $6 \times 10^{13} \mathrm{~W} / \mathrm{cm}^{2}$, which 
corresponds to channel switching from (7+1)-photon ionisation via the 5g Rydberg state to $(8+1)$ photon ionisation via the $5 \mathrm{f}$ Rydberg state [21]. This behaviour will not be elaborated here, but will be the subject of a future publication.

The evolution of the pattern below the first ATI order (typically below $0.5 \mathrm{eV}$, which corresponds to the part of the electron momentum spectra below 0.2 a.u.) is very different from the resonance-enhanced ionisation channel. At the lowest intensity one can observe strong contributions around a momentum of 0.18 a.u. (kinetic energy around $0.45 \mathrm{eV}$ ) at zero and 45 degrees and weaker features at 80 degrees from the vertical laser polarisation axis (see figure 1). Hardly any structure is present at lower energies. Raising the intensity to $4.5 \times 10^{13} \mathrm{~W} / \mathrm{cm}^{2}$ one can see an extension of the observed contributions to lower energies. From here on and up to $8.3 \times 10^{13} \mathrm{~W} / \mathrm{cm}^{2}$ the pattern forming the near-threshold energy region of the momentum maps consists of features fanning out radially from the centre of the image with a constant angular distribution. At $9.5 \times 10^{13} \mathrm{~W} / \mathrm{cm}^{2}$, the highest intensity accessible in our experiment, the radial structure is washed out.

The evolution of the low-energy pattern becomes more dramatic when the laser wavelength is changed. Figure 2 shows a series of momentum maps for wavelengths between 600 and $800 \mathrm{~nm}$ at intensities that were on average $46 \times 10^{13} \mathrm{~W} / \mathrm{cm}^{2}$. In these measurements the ratio of double-to-single ionisation of Xe was used as a rough indicator of the intensity in the measurement, and efforts were taken to keep this ratio as constant as possible. Even so, the variations of the intensities used, as inferred from a comparison of the data with TDSE results that are again shown on the right, were as high as a factor 2. Nevertheless, this disadvantage is offset by the fact that much more significant changes in the low-energy pattern occur in this dataset. These changes are most conveniently illustrated in the column shown to the right of the TDSE momentum maps, where the calculated angular distributions at a photoelectron kinetic energy of $0.25 \mathrm{eV}$ ( $\mathrm{p}=0.135$ a.u.) are shown. One can clearly see how between 600 and $800 \mathrm{~nm}$ the number of minima in the angular distribution changes a number of times, with sometimes a maximum appearing at 90 degrees with respect to the laser polarisation direction, and sometimes a minimum. For example, at $800 \mathrm{~nm}$ the photoelectron angular distribution exhibits 5 minima between 0 and 180 degrees including a minimum at 90 degrees with respect to the laser polarisation axis (figure 2a). Shortening the wavelength to $770 \mathrm{~nm}$, the number of oscillations in the angular distribution changes to 4 and a maximum appears at 90 degrees (figure $2 b$ ). Further decrease of laser wavelength leads once more to 5 minima in the angular distributions at 730 and $700 \mathrm{~nm}$ (figure 2c, 2d) and to 4 minima at the shorter wavelengths (figure $2 \mathrm{e}-2 \mathrm{~g}$ ).

In order to analyze the evolution of the near-threshold pattern with the laser wavelength and intensity, the good qualitative agreement between the experimental and theoretical results will be exploited and we will perform our analysis on the basis of computational results. Specifically, we will monitor the number of minima in the calculated angular distributions as a function of laser wavelength and intensity. Figure 3a shows a contour plot, where the number of minima in the angular distribution 
of Xe between 0 and 180 degrees with respect to the vertical polarisation axis at a fixed electron kinetic energy of $0.25 \mathrm{eV}$ (momentum around 0.14 a.u.) is presented as a function of the laser wavelength and the maximum of the vector potential $\mathrm{A}_{\max }$. The number of minima is encoded in the colour scale. The solid lines highlight the borders for channel closings/openings and are calculated as

$$
\omega=\left(\mathrm{IP}+\mathrm{U}_{\mathrm{p}}\right) / \mathrm{n},
$$

where IP $=0.446$ a.u. is the ionisation potential of $\mathrm{Xe}, \mathrm{U}_{\mathrm{p}}=\mathrm{I}_{0} / 4 \omega^{2}$ the ponderomotive energy of electrons in a laser field with peak intensity $\mathrm{I}_{0}$ and frequency ?, and $\mathrm{n}$ is the number of photons required to reach the ponderomotively shifted ionisation threshold from the ground state. In figure $3 a n=7$ for the uppermost curve and $n=17$ for the lowermost curve. The parabolic shape of the curves is related to the quadratic dependence of the ponderomotive shift on the vector potential $\mathrm{U}_{\mathrm{p}}=\mathrm{A}_{\max }^{2} / 4$. A clear correlation between the channel closings/openings and the change in the number of minima in the angular distribution can be concluded from the contour plot. The parameter range of the experimental data presented in figures 1 and 2 is shown with white and black diamonds, respectively. As observed in the experiment the photoelectron angular distributions of the near-threshold pattern demonstrate 5 minima for all measurements at $700 \mathrm{~nm}$ and either 4 or 5 minima for the measurements between 800 and $600 \mathrm{~nm}$. In connection with figure 1, the contour plot in figure 3 a shows 5 minima at $700 \mathrm{~nm}$ for $\mathrm{A}_{\max }$ between 0.55 and 0.75 a.u. This intensity range is bracketed by the 8-photon channel closing at $\mathrm{A}_{\max }=0.55$ a.u. and closing of the 9-photon channel at $\mathrm{A}_{\max }=0.75$ a.u. Interestingly, both in the experimental and the calculated momentum maps the structure in the near-threshold region is very well-resolved within the 9-photon channel intensity range and becomes somewhat diffuse just beyond that range at $\mathrm{A}_{\max }=0.48$ a.u. and $\mathrm{A}_{\max }=0.8$ a.u. (see figure 1), where according to the contour plot the transitions to the 8-photon and 10-photon channels take place. In connection with the measurements shown in figure 2 , the contour plot in figure 3 a clearly shows that the modulation of the angular distributions in the wavelength-dependent experimental data is connected to channel closings. Accordingly, 5 minima are observed in the angular distribution at $800 \mathrm{~nm}$ from 11-photon ionisation. This is followed by 4 minima at $770 \mathrm{~nm}$ (part of the 10-photon channel), and this is followed by 5 minima at 730 and $700 \mathrm{~nm}$, as part of the 9-photon channel. The appearance of low-energy photoelectrons at a channel closing was previously observed and described by Schyja et al. [22]. In their experiment the observation of the near-threshold pattern was related to the 9-photon channel closing at $800 \mathrm{~nm}$ as the intensity increased from $1.2 \times 10^{13} \mathrm{~W} / \mathrm{cm}^{2}$ to $4 \times 10^{13} \mathrm{~W} / \mathrm{cm}^{2}$. The 9-photon channel closing is expected at $3 \times 10^{13} \mathrm{~W} / \mathrm{cm}^{2}\left(\mathrm{~A}_{\max }=0.52\right.$ a. u.) at $800 \mathrm{~nm}$. According to the contour plot 6 minima are then expected in the angular distribution, which is in full agreement with the angular distribution presented in [22]. 
Remarkably, the correlation between the number of minima in the angular distributions of the near-threshold pattern and the conditions for channel closing/opening is much worse at high values of $\mathrm{A}_{\max }$ for laser frequencies above 0.06 a.u. The breakdown of the correlation persists at even higher intensities as we have observed in TDSE calculations for Ar (figure 3b). Analogous to figure 3a, the solid lines indicating channel closings/openings in figure $3 \mathrm{~b}$ are calculated using Eqn. (1), where $\mathrm{IP}=0.579$ a.u. is the ionisation potential for Ar. In this case, $n=7$ for the uppermost curve and $n=17$ for the lowermost curve. At $A_{\max }<1$ a.u. a correlation between the channel closings/openings and changes in the number of minima in the angular distribution is indicated in the contour plot. At higher intensities however $\left(\mathrm{A}_{\max }>1\right.$ a.u.) this correlation breaks down and the angular distributions become independent of the maximum of the vector potential. Invariance of photoelectron momentum spectra with intensity was previously observed by Maharjan et al. [3]. In their experiment the photoelectron momentum spectra measured in $\mathrm{Ar}$ at $640 \mathrm{~nm}$ show almost identical patterns at intensities of $7.08 \times 10^{14}$ $\mathrm{W} / \mathrm{cm}^{2}$ and $8.2 \times 10^{14} \mathrm{~W} / \mathrm{cm}^{2}$. The TDSE simulations performed by Morishita et al. 23] explain this observation by taking into account the effect of ground state depletion and the laser focal volume averaging. Inspecting the differential intensity contributions to the low-energy part of the photoelectron spectrum Morishita et al. demonstrate that major contributions to the ionisation yield come from relatively low intensities around $2 \times 10^{14} \mathrm{~W} / \mathrm{cm}^{2}\left(\mathrm{~A}_{\max }=1.1\right.$ a.u. $)$ that are present in the focal volume. Consistent with this explanation, the angular distributions in our calculations of the nearthreshold pattern for Ar remain unchanged at $A_{\max }>1$ a.u. (figure 3b). We note that the near-threshold pattern experimentally observed in Ar at $640 \mathrm{~nm}$ and at various intensities in [3] and calculated under the same conditions in [23] is well reproduced in our calculations (not presented here).

Until recently, the origin of the angular distribution of near-threshold photoelectrons has remained an open question. In their theoretical study of photoelectron momentum spectra in Argon, Neon, Helium and Hydrogen, Chen et al. [6] established a relation of the radial pattern to the longrange Coulomb potential of the parent ion and suggested an empirical rule for the prediction of the dominant angular momentum of the near-threshold photoelectrons. According to their model the number of minima in the angular distributions near threshold depends only on the number of photons absorbed and does not change until the next channel closing. Very recently Arbó et al. [8] have derived a semi-classical expression for the dominant angular momentum of the emitted electrons, whose classical trajectories interfere in the presence of both the Coulomb and the laser fields. Providing a quantitative agreement with the empirical relation proposed by Chen et al. [6], the analytical formula by Arbó et al. shows that the near-threshold electron angular distribution depends on the laser field amplitude and frequency, or alternatively, on the field amplitude, ionisation potential and the minimum number of photons needed to reach the continuum.

The semi-classical prediction by Arbó et al. has shown good quantitative agreement with the previously published results of TDSE calculations for various atomic species. In figure 4 we compare the results given by the analytical formula derived by Arbó et al. with the results of our experiment 
and TDSE calculations for Xe and Ar. According to [8] the dominant classical angular momentum $L$ of photoelectrons with close-to-zero final kinetic energy can be expressed in the following way:

$$
L=\left(2 Z_{T} \alpha\right)^{1 / 2},
$$

where $Z_{T}$ is the asymptotic charge of the atomic potential and $\alpha=\mathrm{A}_{\max } / \omega=\mathrm{F}_{0} / \omega^{2}$ is the electron quiver amplitude in a laser field with amplitude $\mathrm{F}_{0}$, frequency $\omega$ and a maximum of the vector potential $\mathrm{A}_{\max }$. As emphasised in [8], the prediction of the classical angular momentum given by Eqn. (2) is valid within the uncertainty $L \pm 1$ due to quantum discreteness. In figure 4 a the semi-classical prediction of the number of minima is shown with a solid line as a function of the maximum of the vector potential $\mathrm{A}_{\max }$ at a constant laser frequency $\omega=0.065$ a.u. $(\lambda=700 \mathrm{~nm}$ ). Our experimental data for Xe (shown with diamonds) fit very well with the semi-classical prediction within the quantum 'binning' uncertainty (shown with dashed lines). We also present the results of our TDSE calculations for Xe and $\mathrm{Ar}$ at $700 \mathrm{~nm}$ for $\mathrm{A}_{\max }=0.45-1.0$ a.u. and $\mathrm{A}_{\max }=0.45-1.8$ a.u, respectively. In agreement with the semi-classical prediction, we observe an overall increase of the dominant angular momentum with the maximum of the vector potential. In addition, the TDSE results clearly demonstrate a modulation of the angular momentum with the occurrence of channel closings as the vector potential grows. In figure 4a the channel closing events for Ar and Xe are marked with vertical bars in the top (Ar, blue) and in the bottom (Xe, red) parts of the graph, respectively. The labels at the vertical bars correspond to the minimum number of photons required to reach the ionisation threshold. The quantitative agreement of the TDSE results with the semi-classical prediction is observed over a large range of $\mathrm{A}_{\max }$ for both $\mathrm{Ar}$ and $\mathrm{Xe}$ and confirms the universal character of the electron dominant angular momentum near ionisation threshold, independent of the atomic properties.

Further inspection of figure $4 \mathrm{a}$ reveals deviations of the TDSE results from the semi-classical prediction at high values of $\mathrm{A}_{\max }$. The invariance of the electron angular distributions at relatively high intensities that we discussed above in relation to figure 3, leads to a departure from the semi-classical model. This can be clearly observed in figure $4 \mathrm{~b}$, where the classical angular momentum is compared to the TDSE results for Ar at several laser wavelengths. Note that plotting the dominant angular momentum against the electron quiver amplitude eliminates the dependence of the semi-classical prediction on the laser frequency according to Eqn. (2). Figure $4 \mathrm{~b}$ shows that for a given laser frequency, there is a certain quiver amplitude, or alternatively, laser intensity, beyond which the dominant angular momentum remains unchanged and eventually leaves the uncertainty range of the semi-classical prediction. This observation sets a limitation for the semi-classical model and should be taken into account for comparison with experimental results at high intensities.

\section{Conclusion}


In conclusion, we have performed an experimental and theoretical study of the photoelectron properties in near-threshold atomic ionisation. We have experimentally observed that the evolution of the electron angular distribution near the ionisation threshold is not sensitive to the channel switching effect and therefore, not related to resonant ionisation. Rather, our experiment provides quantitative agreement with the semi-classical model of Arbó and co-workers [8], which relates the origin of the oscillatory angular distribution of slow photoelectrons to interfering classical electron trajectories launched at different times during the laser pulse. Systematic analysis of the near-threshold pattern in our TDSE calculations as a function of the laser frequency and the maximum of the vector potential has demonstrated a correlation of the dominant angular momentum of the electron with channel closing/opening effects over a wide range of parameters. Our TDSE calculations predict a breakdown of this correlation upon further increase of laser intensity.

\section{Acknowledgement}

This work is part of the research program of the "Stichting voor Fundamenteel Onderzoek der Materie" (FOM), which is financially supported by the "Nederlandse Organisatie voor Wetenschappelijk Onderzoek" (NWO). KJS is supported by National Science Foundation Grant No. PHY-0701372. 


\section{References}

[1] Helm H, Bjerre N, Dyer M J, Huestis D L and Saeed M 1993 Images of photoelectrons formed in intense laser fields Phys. Rev. Lett. 703221

[2] Rudenko A, Zrost K, Schröter C D, de Jesus V L B, Feuerstein B, Moshammer R and Ullrich J 2004 Resonant structures in the low-energy electron continuum for single ionization of atoms in the tunnelling regime J. Phys. B 37 L407

[3] Maharjan C M, Alnaser A S, Litvinyuk I, Ranitovic P and Cocke C L 2006 Wavelength dependence of momentum-space images of low-energy electrons generated by short intense laser pulses at high intensities J. Phys. B 391955

[4] Arbó D G, Yoshida S, Persson E, Dimitriou K I and Burgdörfer J 2006 Interference oscillations in the angular distribution of laser-ionized electrons near ionization threshold Phys. Rev. Lett. 96143003

[5] Wickenhauser M, Tong X M, Arbó D G, Burgdörfer J and Lin C D 2006 Signatures of tunneling and multiphoton ionization in the electron-momentum distributions of atoms by intense few-cycle laser pulses Phys. Rev. A $\mathbf{7 4} 041402$

[6] Chen Z, Morishita T, Le A T, Wickenhauser M, Tong X M and Lin C D 2006 Analysis of twodimensional photoelectron momentum spectra and the effect of the long-range Coulomb potential in single ionization of atoms by intense lasers Phys. Rev. A 74053405

[7] Arbó D G, Miraglia J E, Gravielle M S, Schiessl K, Persson E and Burgdörfer J 2008 CoulombVolkov approximation for near-threshold ionization by short laser pulses Phys. Rev. A 77013401

[8] Arbó D G, Dimitriou K I, Persson E and Burgdörfer J 2008 Sub-Poissonian angular momentum distribution near threshold in atomic ionization by short laser pulses Phys. Rev. A 78013406

[9] Dionissopoulou S, Mercouris Th, Lyras A and Nicolaides C A 1997 Strong laser-filed effects in hydrogen: High-order above-threshold ionization and photoelectron angular distributions Phys. Rev. A $\mathbf{5 5} 4397$

[10] Wiehle R, Witzel B, Helm H and Cormier E 2003 Dynamics of strong-field above-threshold ionization of argon: comparison between experiment and theory Phys. Rev. A 67063405

[11] Eppink A T J and Parker D H 1997 Velocity map imaging of ions and electrons using electrostatic lenses: Application in photoelectron and photofragment ion imaging of molecular oxygen Rev. Sci. Instrum. 683477

[ 12] Vrakking M J J 2001 An iterative procedure for the inversion of two-dimensional ion/photoelectron imaging experiments Rev. Sci. Instrum. 724084

[13] Kulander K C, Schafer K J and Krause J L 1992 Atoms in Intense Laser Fields, ed MGavrila (New York: Academic Press)

[14] Schafer K J and Kulander K C 1990 Energy analysis of time-dependent wave functions: application to above-threshold ionization Phys. Rev. A 425794

[15] Schafer K J 2008 Numerical methods in strong field physics, in Strong Field Laser Physics, ed T Brabec (New York: Springer Science + Business Media) 
[16] Kulander K C and Rescigno T N 1991 Effective potentials for time-dependent calculations of multiphoton processes in atoms Comput. Phys. Commun. 63523

[17] Muller H G 1999 An efficient propagation scheme for the time-dependent Schrödinger equation in the velocity gauge Laser Phys. 9138

[18] Muller H G and Kooiman F C 1998 Bunching and focusing of tunneling wave packets in enhancement of high-order ATI Phys. Rev. Lett. 811207

[19] Schafer K J 1991 The energy analysis of time-dependent numerical wave-functions Comput. Phys. Commun. 63427

[20] Augst S, Meyerhofer D D, Strickland D and Chin S L 1991 Laser ionization of noble gases by Coulomb-barrier suppression J. Opt. Soc. Am. B 8858

[21] NIST atomic database http://physics.nist.gov/PhysRefData/ASD/index.html

[22] Schyja V, Lang T and Helm H 1998 Channel switching in above-threshold ionization of xenon Phys. Rev. A 573692

[23] Morishita T, Chen Z, Watanabe S and Lin C D 2007 Two-dimensional electron momentum spectra of argon ionized by short intense lasers: Comparison of theory with experiment Phys. Rev. A 75023407 


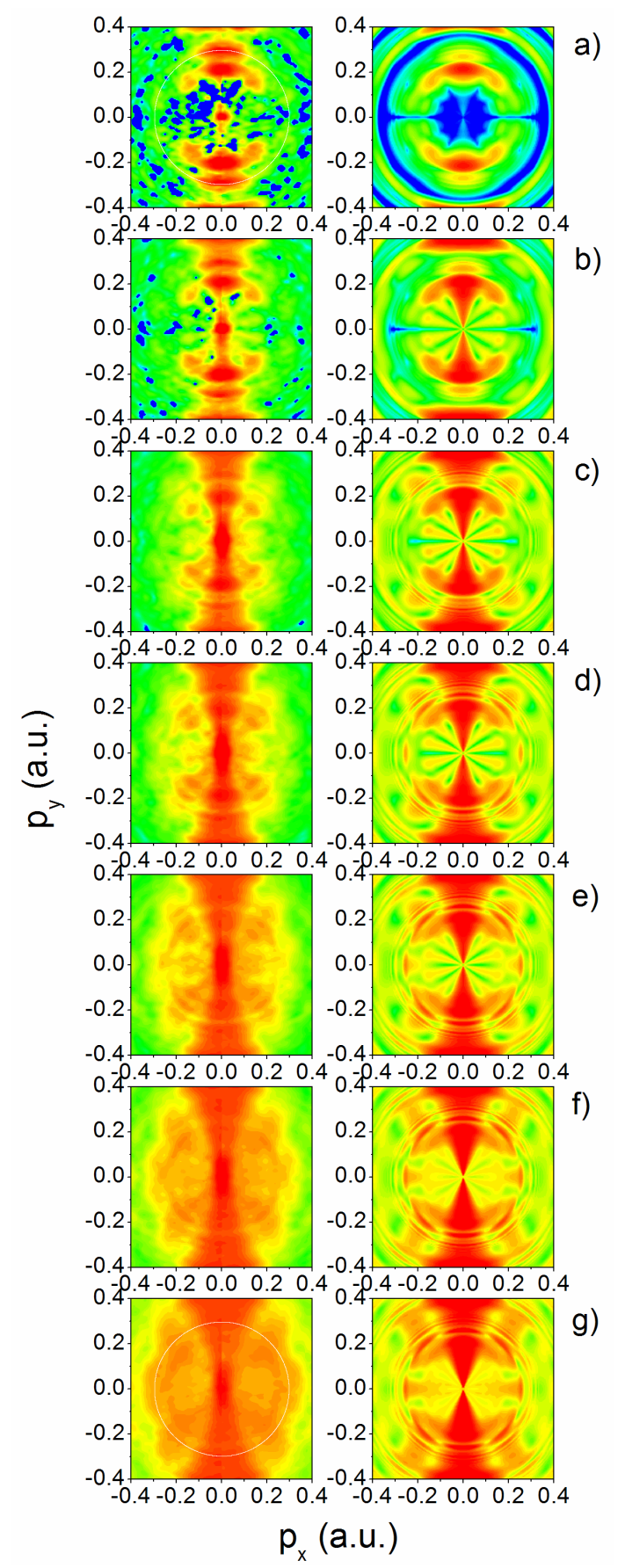

Figure 1. (Colour online) Experimental (left column) and calculated (right column) momentum maps for ionisation of Xe at a wavelength of $700 \mathrm{~nm}$ and at various intensities: a) $\mathrm{E}_{\text {pulse }}=25 \mu \mathrm{J}, \mathrm{A}_{\max }=0.48$ a.u. $\left(3.4 \times 10^{13} \mathrm{~W} / \mathrm{cm}^{2}\right)$; b) $\mathrm{E}_{\text {pulse }}=30 \mu \mathrm{J}, \mathrm{A}_{\max }=0.55$ a.u. $\left.\left(4.5 \times 10^{13} \mathrm{~W} / \mathrm{cm}^{2}\right) ; \mathrm{c}\right) \mathrm{E}_{\mathrm{pulse}}=35 \mu \mathrm{J}, \mathrm{A}_{\max }=0.63$ a.u. $\left(5.9 \times 10^{13} \mathrm{~W} / \mathrm{cm}^{2}\right)$; d) $\mathrm{E}_{\text {pulse }}=40 \mu \mathrm{J}, \mathrm{A}_{\max }=0.66$ a.u. $\left(6.4 \times 10^{13} \mathrm{~W} / \mathrm{cm}^{2}\right)$; e) $\mathrm{E}_{\text {pulse }}=45 \mu \mathrm{J}, \mathrm{A}_{\max }=0.71$ a.u. $\left(7.5 \times 10^{13} \mathrm{~W} / \mathrm{cm}^{2}\right) ;$ f) $\mathrm{E}_{\text {pulse }}=50 \mu \mathrm{J}, \mathrm{A}_{\max }=0.75$ a.u. $\left.\left(8.3 \times 10^{13} \mathrm{~W} / \mathrm{cm}^{2}\right) ; \mathrm{g}\right) \mathrm{E}_{\text {pulse }}=60 \mu \mathrm{J}, \mathrm{A}_{\max }=0.8$ a.u. $\left(9.5 \times 10^{13} \mathrm{~W} / \mathrm{cm}^{2}\right)$. The logarithmic false-colour scale covers 5 orders of magnitude. The white circles show the contribution from electrons corresponding to one-photon ionisation via $5 \mathrm{~g} / 5 \mathrm{f}$ Rydberg state, leading to photoelectrons with a kinetic energy of $1.2 \mathrm{eV}$. 


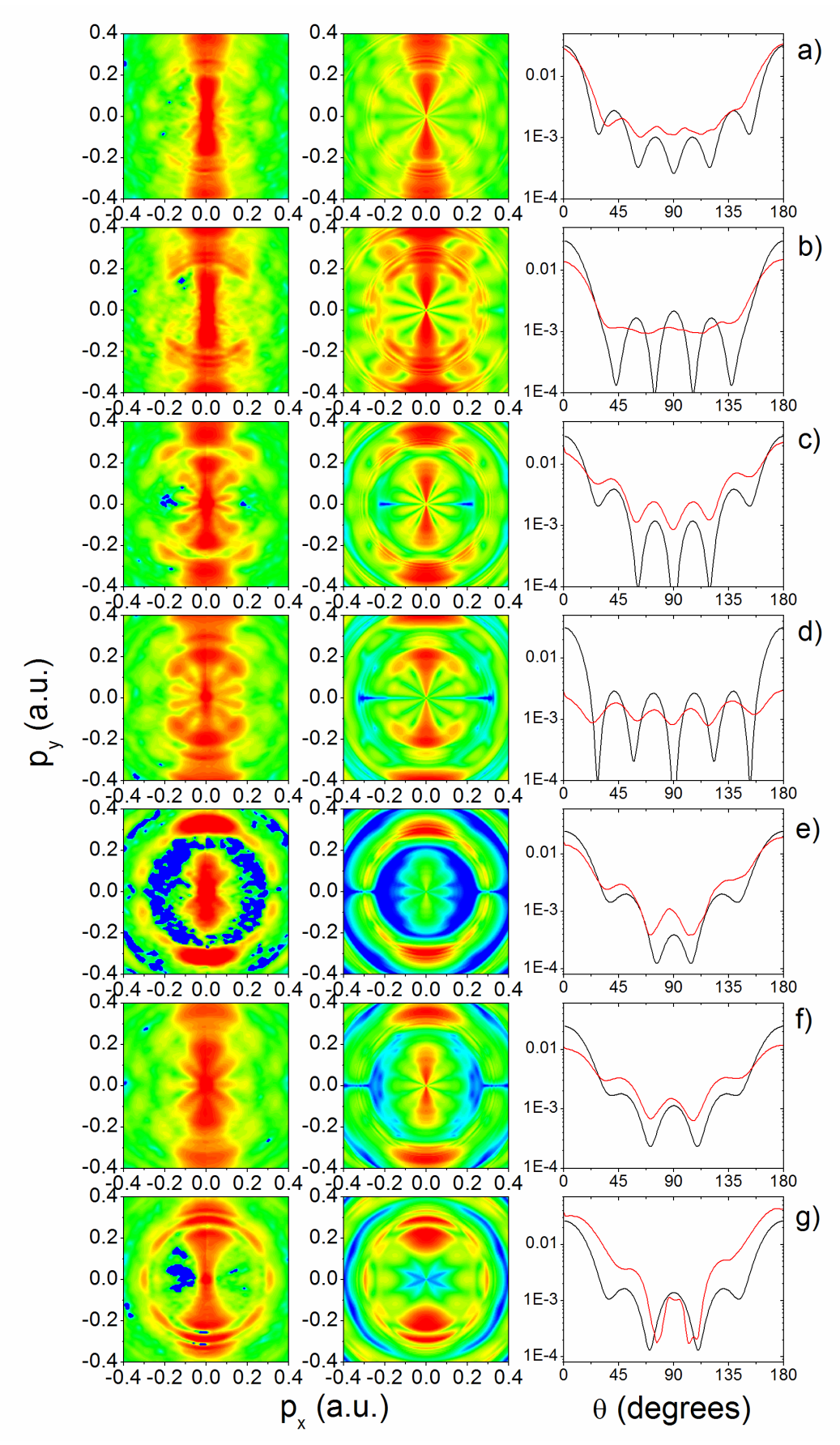

Figure 2. (Colour online) Experimental (left column) and calculated (middle column) momentum maps for ionisation of $\mathrm{Xe}$ at various wavelengths and intensities: a) $800 \mathrm{~nm}, \mathrm{~A}_{\max }=0.77$ a.u. $\left(6.7 \times 10^{13}\right.$ $\left.\mathrm{W} / \mathrm{cm}^{2}\right)$; b) $770 \mathrm{~nm}, \mathrm{~A}_{\max }=0.7$ a.u. $\left(6 \times 10^{13} \mathrm{~W} / \mathrm{cm}^{2}\right)$; c) $730 \mathrm{~nm}, \mathrm{~A}_{\max }=0.54$ a.u. $\left(3.9 \times 10^{13} \mathrm{~W} / \mathrm{cm}^{2}\right)$; d) $700 \mathrm{~nm}, \mathrm{~A}_{\max }=0.55$ a.u. $\left(4.5 \times 10^{13} \mathrm{~W} / \mathrm{cm}^{2}\right)$; e) $680 \mathrm{~nm}, \mathrm{~A}_{\max }=0.45$ a.u. $\left(3.2 \times 10^{13} \mathrm{~W} / \mathrm{cm}^{2}\right)$; f) $650 \mathrm{~nm}$, $\mathrm{A}_{\max }=0.52$ a.u. $\left.\left(4.6 \times 10^{13} \mathrm{~W} / \mathrm{cm}^{2}\right) ; \mathrm{g}\right) 600 \mathrm{~nm}, \mathrm{~A}_{\max }=0.53$ a.u. $\left(5.7 \times 10^{13} \mathrm{~W} / \mathrm{cm}^{2}\right)$. The logarithmic falsecolour scale covers 4 orders of magnitude. The right column shows experimental and calculated angular distributions at a photoele ctron kinetic energy of $0.25 \mathrm{eV}$ ( $\mathrm{p}=0.135$ a.u.). 

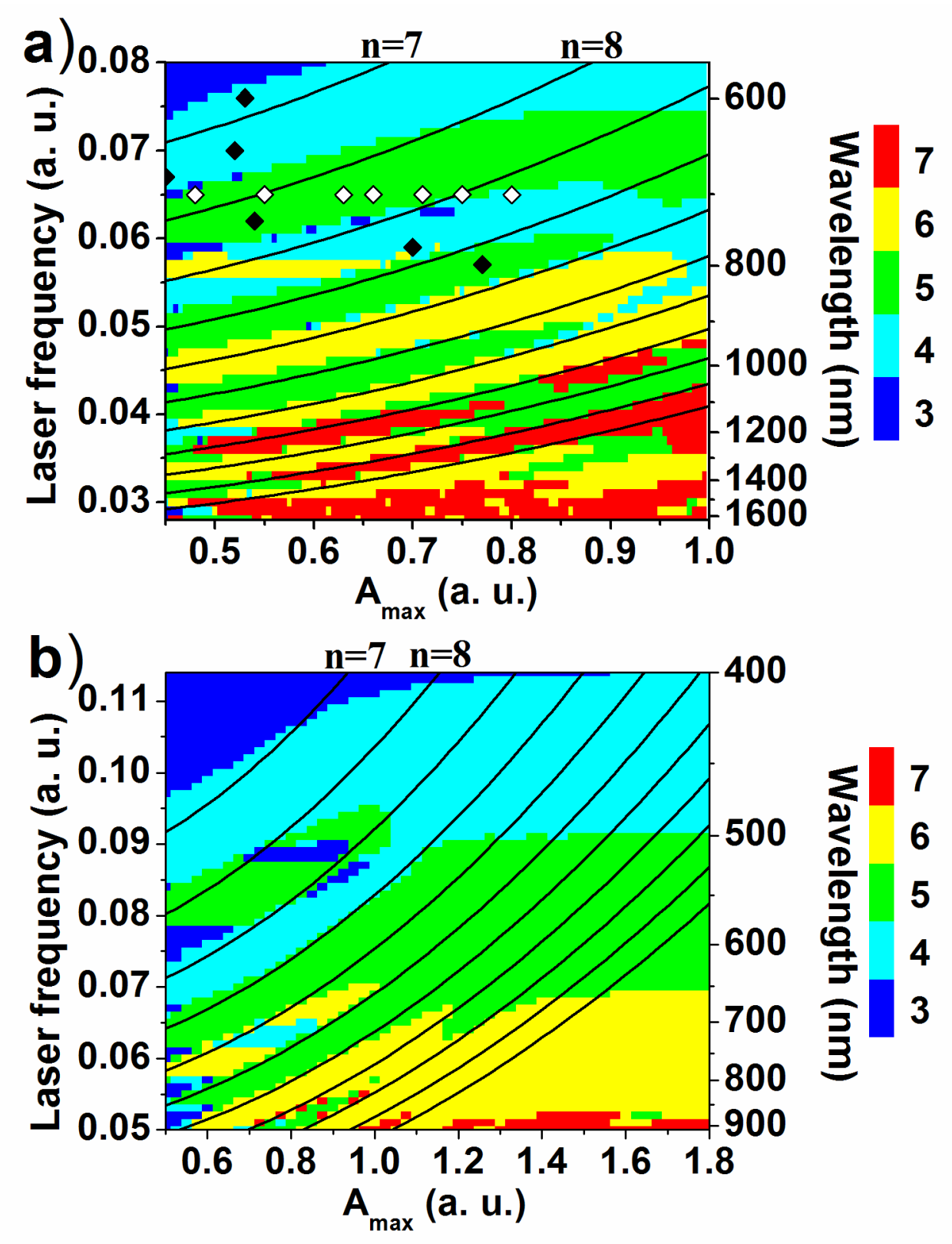

Figure 3. (Colour online) Contour plots showing the number of minima between 0 and 180 degrees in the angular distributions in a) $\mathrm{Xe}$ and b) Ar, calculated at the photoelectron kinetic energy $0.25 \mathrm{eV}$ as a function of the laser wavelength and the maximum of the vector potential. The solid lines show the borders for channel openings/closings, where the uppermost and lowermost curves correspond to 7photon and 17-photon channel closing, respectively (see labels for the top curves). White and black diamonds in a) show parameters range of the experimental data presented in figures 1 and 2 , respectively. 

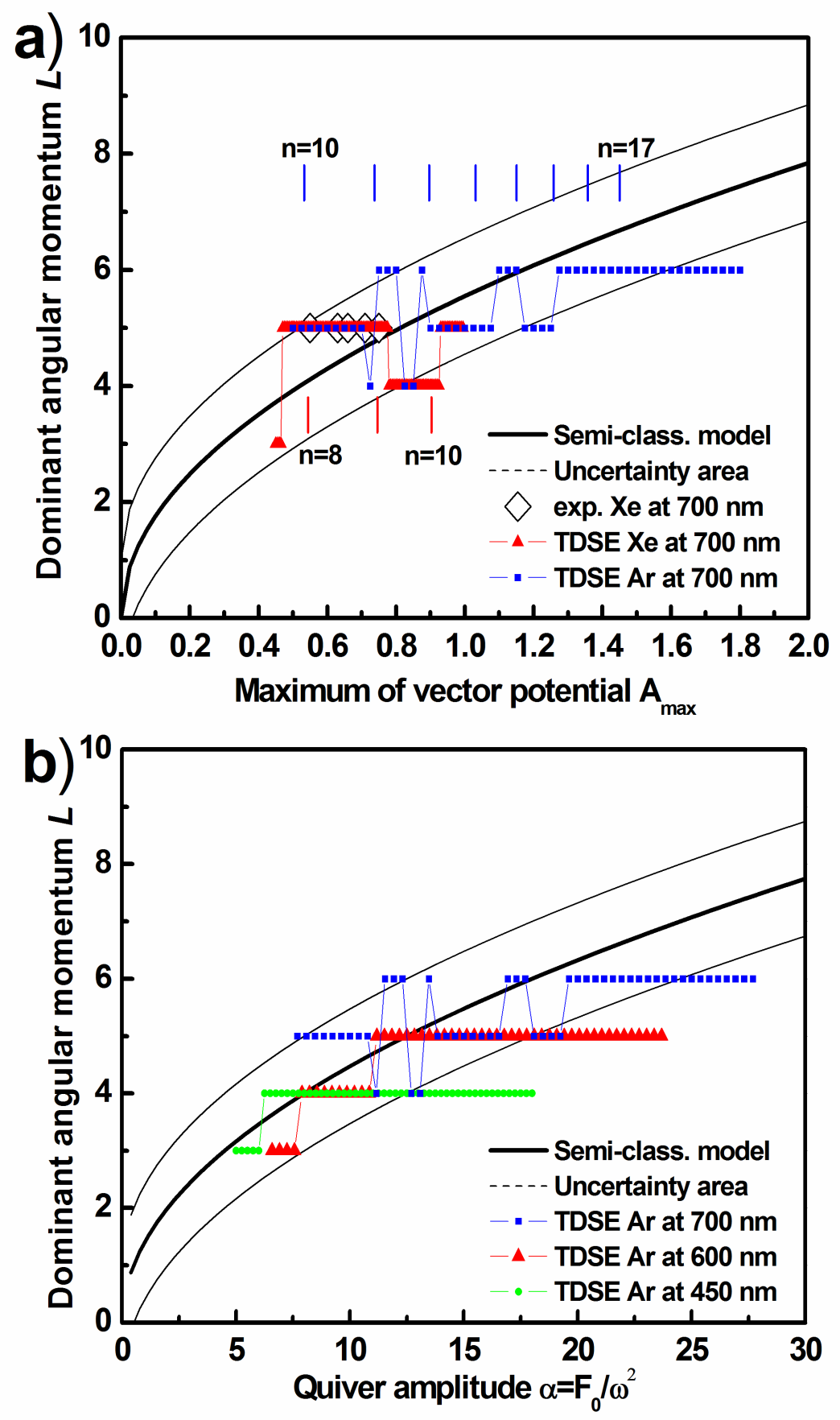

Figure 4. (Colour online) a) Dominant angular momentum as a function of the maximum of the vector potential $\mathrm{A}_{\max }$ at the laser wavelength $\lambda=700 \mathrm{~nm}$. Diamonds - experimental data. Triangles and squares - TDSE for Xe and Ar, respectively. Vertical bars in the top and in the bottom parts of the graph show channel closing events for Ar and Xe, respectively. Solid line - semi-classical prediction [Eqn. (2)], dashed lines limit the uncertainty area due to quantum discreteness. b) Dominant angular momentum as a function of the quiver amplitude $\alpha$. Squares, triangles and circles are TDSE calculations for Ar at 700, 600 and $450 \mathrm{~nm}$ laser wavelength, respectively. Solid line - semi-classical prediction [Eqn. (2)], dashed lines limit the uncertainty area due to quantum discreteness. 